\title{
Anti-proliferative effect of Jesridonin on paclitaxel-resistant EC109 human esophageal carcinoma cells
}

\author{
CONG WANG $^{1-3^{*}}$, LIUBIN GUO $^{4^{*}}$, SAIQI WANG ${ }^{1-3}$, JUNWEI WANG $^{1-3}$, YONGMEI LI $^{1-3}$, \\ YINHUI DOU ${ }^{1-3}$, RAN WANG $^{1-3}$, HONGGE SHI ${ }^{1-3}$, YU KE $^{1-3}$ and HONGMIN LIU ${ }^{1-3}$ \\ ${ }^{1}$ Key Laboratory of Technology of Drug Preparation (Zhengzhou University), Ministry of Education of China; \\ ${ }^{2}$ Collaborative Innovation Center of New Drug Research and Safety Evaluation; ${ }^{3}$ School of Pharmaceutical Sciences, \\ Zhengzhou University, Zhengzhou, Henan 450001; ${ }^{4}$ Department of Basic Medicine, School of Basic Medicine \\ and Clinical Pharmacy, China Pharmaceutical University, Nanjing, Jiangsu 210009, P.R. China
}

Received December 15, 2015; Accepted January 13, 2017

DOI: $10.3892 /$ ijmm.2017.2867

\begin{abstract}
Chemoresistance to anticancer drugs is a major obstacle in the efforts to develop a successful treatment strategy for esophageal squamous carcinoma (ESCC). Thus, the exploration of new drugs and treatment strategies for combating resistance are of utmost importance. In this study, we investigated the antitumor drug resistance activity of Jesridonin, a new ent-kaurene diterpenoid, and its possible mechanisms of action using the resistant cancer cell line, EC109/Taxol. MTT assay revealed that Jesridonin had similar $\mathrm{IC}_{50}$ values against EC109 paclitaxel-sensitive cells and drug-resistant EC109/Taxol cells in vitro. In mice, Jesridonin effectively prevented the growth of EC109/Taxol tumor xenografts without exerting any significant toxicity. In addition, Jesridonin significantly inhibited the proliferation of EC109/Taxol cells, induced apoptosis and arrested the cell cycle at the G2/M phase. Furthermore, western blot analysis revealed that Jesridonin upregulated the expression of $\mathrm{p} 53$, p53 upregulated modulator of apoptosis (PUMA), cleavedcaspase-9 and cleaved-caspase-3 in EC109/Taxol cells, and downregulated the expression of procaspase-3, procaspase- 9 and Bcl-2 in the EC109/Taxol cells in a concentrationdependent manner. Overall, our results demonstrate that Jesridonin may have potential for use in the treatment of paclitaxel-resistant ESCC. The data of the present study may lead to the development of novel treatment strategies for paclitaxel-resistant tumors.
\end{abstract}

Correspondence to: Professor Hongmin Liu, Key Laboratory of Technology of Drug Preparation (Zhengzhou University), Ministry of Education of China, 100 Kexue Avenue, Zhengzhou, Henan 450001, P.R. China

E-mail: liuhm@zzu.edu.cn

*Contributed equally

Key words: multidrug resistance, Jesridonin, apoptosis, mechanism

\section{Introduction}

Chemotherapy is one of the main therapeutic strategies in the treatment of cancer. However, the success of chemotherapy depends on the sensitivity of the tumor to the anti-neoplastic agent (1). Cancer cells can develop resistance to the anticancer drugs used during cancer therapy (2). The drug resistance properties of the tumor greatly limit the effectiveness of chemotherapeutic drugs, resulting in the failure of chemotherapy and the recurrence of the tumor. Furthermore, cancer cells exposed to one drug may exhibit cross-resistance to other drugs that have different structures and functions. This phenomenon is known as multidrug resistance (MDR) and can be intrinsic or acquired following chemotherapy using a cytotoxic agent $(3,4)$. Resistance to anticancer drugs is a major obstacle for successful chemotherapy. Thus, the exploration of new drugs and strategies with which to combat drug resistance is of utmost importance.

Paclitaxel (PTX) is frequently used in all clinical chemotherapy protocols and is an important drug for the treatment of esophageal carcinoma; however, resistance has been observed in clinical practice. Resistance to PTX has been attributed to several mechanisms, including the overexpression of the P-gp efflux pump and resistance to apoptosis, among others $(5,6)$. Consequently, these mechanisms force cells to dysregulate apoptosis, which facilitates tumor progression. The majority of chemotherapeutic drugs kill cancer cells via the induction of apoptosis; however, cancer cells can escape apoptosis or antagonize apoptosis. Some scientists believe that cells with the MDR characteristic often exhibit resistance to apoptosis, and resistance to apoptosis may be one of the essential characteristics of MDR $(4,7,8)$.

Isodon rubescens, a Chinese herb, has been used as a folk botanical medicine in China for the treatment of cancer and inflammatory diseases for many years. Oridonin, an active diterpenoid compound found in Isodon rubescens, has been widely used in the treatment of human diseases ranging from inflammation to cancer $(9,10)$. Jesridonin (JD; chemical structure shown in Fig. 1) is a diterpenoid compound that was obtained via the structural modification of oridonin. Recently, in a previous study, we demonstrated that JD exhibited strong antitumor activity in EC109 human esophageal carcinoma 
cells, both in vitro and in vivo, and demonstrated no adverse effects on major organs in nude mice (11). However, the anti-tumor activity of JD in paclitaxel-resistant cells remains unclear. To overcome the obstacles related to drug resistance and to improve the clinical outcomes of patients with esophageal squamous carcinoma (ESCC), in this study, we examined the effects of JD on the PTX-resistant EC109 human cancer cell line, EC109/Taxol, with the ultimate goal of identifying a novel therapy for paclitaxel-resistant ESCC.

\section{Materials and methods}

Reagents. Propidium iodide (PI), dimethyl sulfoxide (DMSO), 3-(4,5-dimethylthiazol-2-yl)-2,5-diphenyltetrazolium bromide (MTT) and bisbenzimide Hoechst 33258 were from Sigma (St.Louis, MO, USA). RNase A and the bicinchoninic acid (BCA) protein assay kit were from Solarbio (Beijing, China). The rabbit monoclonal antibodies against p53 (sc-393031), caspase-9 (sc-8355) and caspase-3 (sc-65497) are the products of Santa Cruz Biotechnology, Inc. (Santa Cruz, CA, USA). Rabbit monoclonal antibodies against Bcl-2 (ZA-0536) and Bax (TA337090) were from Zhongshan Goldenbridge Biotechnology, Co., Ltd. (Beijing, China). Primary antibody against p53 upregulated modulator of apoptosis (PUMA; E1A5173) was from Nanjing EnoGene Biotechnology (Nanjing, China). Primary antibody against cleaved caspase-3 (\#9661) and cleaved caspase-9 (\#9501) was purchased from Cell Signaling Technology (Danvers, MA, USA). Peroxidase-conjugated affinipure goat anti-rabbit IgG (ZB-2301) and anti-mouse IgG (ZB-2305) IgG as secondary antibodies were from Zhongshan Goldenbridge Biotechnology, Co., Ltd.

Cell culture and compounds. The EC109 cell line was obtained from the Chinese Academy of Sciences (Shanghai, China). The cells were cultured in RPMI-1640 medium supplemented with $10 \%$ fetal bovine serum (FBS) (both from HyClone, Beijing, China), $100 \mathrm{U} / \mathrm{ml}$ of penicillin and $100 \mu \mathrm{g} / \mathrm{ml}$ of streptomycin at $37^{\circ} \mathrm{C}$ in a humidified air atmosphere containing $5 \% \mathrm{CO}_{2}$. The resistant cell line (EC109/Taxol) was developed in vitro by the intermittent exposure of the human ESCC cell line, EC109, to a high concentration of PTX, with a stepwise increase in concentration over a period of 6 months. These cells were developed, cultured and maintained as previously described (8).

JD was obtained from the New Drug Research and Development Center of Zhengzhou University. JD is a 7,14-acetal derivative of Oridonin (a natural antitumor compound isolated from Isodon rubescens). The chemical structure was confirmed using NMR, MS and IR data (Fig. 1). For the in vitro assay, JD was dissolved in DMSO and stored at $-20^{\circ} \mathrm{C}$. The concentration of DMSO in the culture medium was under $1 \%$ (v/v) and had no intrinsic effect on cell proliferation.

Animals. A total of 15 female 6-week-old BALB/c nu/nu mice weighing 18-19 g each were purchased from Hunan Slack King of Laboratory Animal, Co., Ltd. (Hunan, China) and maintained under specific pathogen-free (SPF) conditions at $25^{\circ} \mathrm{C}$ in an atmosphere with $50 \%$ humidity for the experiments. Lighting was operated automatically on a 12-h light/ dark cycle. The mice were raised in a sterile environment and received adequate water and food. Throughout the trial period, all experiments strictly followed institutional guidelines and were approved by the Experimental Animal Care Committee of Zhengzhou University (approval no. SPS140302).

Cytotoxic activity assays. The cells $\left(8 \times 10^{3}\right.$ cells/well $)$ were inoculated into each well in 96-well plates (Nest Biotechnology Co., Ltd., Wuxi, Jiangsu, China) in $100 \mu \mathrm{l}$ of culture medium. Following an overnight incubation $\left(37^{\circ} \mathrm{C}\right.$ with $5 \% \mathrm{CO}_{2}$ ), the medium was removed and replaced with various concentrations of JD. The plates were incubated at $37^{\circ} \mathrm{C}$ in $5 \% \mathrm{CO}_{2}$ for 24,48 and $72 \mathrm{~h}$. Thereafter, $20 \mu \mathrm{l}$ of MTT solution $(5 \mathrm{mg} / \mathrm{ml})$ were added to each well, and the plate was incubated for an additional $4 \mathrm{~h}$ at $37^{\circ} \mathrm{C}$. The resulting formazan crystals were dissolved in $150 \mu \mathrm{l}$ of DMSO following aspiration of the culture medium. The plates were read at $490 \mathrm{~nm}$ using a microplate reader (BioTek Instruments, Inc., Winooski, VT, USA). The 50\% inhibitory concentration $\left(\mathrm{IC}_{50}\right)$ represents the concentration of the modulators that was required for $50 \%$ inhibition.

Cell proliferation assay. The cells were seeded at $1.4 \times 10^{4}$ cells/well (in 24 -well plates). Following an overnight incubation, the medium was removed and replaced by $10 \mu \mathrm{M}$ of JD. Cells from 3 wells were trypsinized and separately counted at the time points of 24,48,72,96,120, 144 and $168 \mathrm{~h}$ following treatment with JD.

Colony formation assay. A colony formation assay was performed to determine the effects of JD treatment on the colony-forming ability of EC109/Taxol cells. Briefly, the cells were seeded into 6 -well plates at $1 \times 10^{3}$ cells/well. Following an overnight incubation, the medium was removed and replaced by various concentrations of JD. Following incubation for 7 days, the cells were then fixed with $95 \%$ ethanol for $30 \mathrm{~min}$ and stained with $0.5 \%$ crystal violet for $30 \mathrm{~min}$. Cell colonies ( $\geq 50$ cells) were counted under an inverted light microscope (BDS15010071, Aote light microscope, Chongqing, China). The colony formation rate was calculated as follows: (colony counts)/(cells inoculated) x100\%.

Assessment of cancer growth in mice. The evaluation of JD activity was also carried out on nude mice bearing EC109/ Taxol xenografts. The tumor model was established as previously described (8) by subcutaneously inoculating EC109/Taxol cells $\left(1 \times 10^{7} /\right.$ mice $)$ into the left armpits of the mice. The tumors were allowed to reach an average size of $100-150 \mathrm{~mm}^{3}$ (as estimated by Vernier caliper measurement), and the mice bearing the EC109/Taxol cells were then randomly divided into 3 groups. The 3 groups of mice were treated as follows: the mice in group $\mathrm{C}$ were the free control, the mice in group $\mathrm{T} 1$ were treated with $5 \mathrm{mg} / \mathrm{kg}$ of JD, and the mice in group T2 were treated with $10 \mathrm{mg} / \mathrm{kg}$ of JD ( $\mathrm{n}=5 \mathrm{mice} / \mathrm{group})$. The drug (the clathrate of JD was formed with $\beta$-cycloamylose and dissolved in $0.9 \% \mathrm{NaCl})$ and the vehicle $(\beta$-cycloamylose in $0.9 \% \mathrm{NaCl}$, $10 \mathrm{mg} / \mathrm{kg}$ ) were administered by intravenous tail vein injection every 3 days for a total of 21 days. At the end of the treatment, the mice were sacrificed by cervical vertebrae dislocation, and the tumors were dissected and weighed. During this period, the body weights of the animals were recorded every 3 days, and the tumor sizes were measured by determining 2 perpendicular 
Table I. The $\mathrm{IC}_{50}$ values of JD on EC109 and EC109/Taxol cells.

\begin{tabular}{lcc}
\hline & \multicolumn{2}{c}{$\mathrm{IC}_{50}(\mu \mathrm{M})$} \\
\cline { 2 - 3 } Time (h) & \multicolumn{1}{c}{$\mathrm{EC} 109$} & $\mathrm{EC} 109 /$ Taxol \\
\hline 24 & $24.354 \pm 0.267$ & $25.679 \pm 2.505$ \\
48 & $14.470 \pm 0.518$ & $19.829 \pm 0.090^{\mathrm{a}}$ \\
72 & $8.590 \pm 0.467$ & $6.684 \pm 0.420^{\mathrm{a}}$
\end{tabular}

Data are the means $\pm \mathrm{SD}$ of at least 3 different experiments. ${ }^{\mathrm{a}} \mathrm{P}<0.05$ vs. EC109 cell group. JD, Jesridonin.

dimensions at the same time. The volume (V) of each tumor was then calculated using the following formula:

$$
V=a x b^{2} x 1 / 2
$$

where length (a, mm) is the longest diameter and width (b, mm) is the shortest diameter perpendicular to the length $(12,13)$. The calculated tumor volumes were expressed in percentages as relative tumor volume (RTV) using the following equation (14):

$$
\mathrm{RTV}=\mathrm{V}_{\mathrm{n}} / \mathrm{V}_{0}
$$

where $\mathrm{V}_{\mathrm{n}}$ is the tumor volume at day $\mathrm{n}$ of treatment and $\mathrm{V}_{0}$ represents the initial tumor volume at the onset of treatment. The inhibition of cancer growth was defined as a ratio of the tumor weight compared to the vehicle control tumor weight. The specimens from the EC109/Taxol xenografts were removed for hematoxylin and eosin (H\&E) staining.

$H \& E$ staining assay. The specimens of EC109/Taxol xenografts were fixed in $10 \%$ formalin and then processed by the usual technique of paraffin inclusion. The histological technique of paraffin inclusion used in this study was performed in the following sequence: dehydration, clarification, waxing and proper inclusion. The embedded tissues were cut into 4- $\mu$ m-thick sections, affixed to glass slides, deparaffinized in xylene, rehydrated in ethanol, rinsed in distilled water and then fixed with $4 \%$ formaldehyde. Thereafter, the paraffin sections were stained with $H \& E$, dehydrated in graded alcohol, cleared in xylene, cemented with neutral resin. General morphologies were viewed under a light microscope (347655; Leica Microsystems CMS GmbH, Wetzlar, Germany).

Nuclear staining by Hoechst 33258 . The cells were seeded in a 6-well plate and treated with JD at the indicated concentrations for $24 \mathrm{~h}$. The cells were then washed with phosphate-buffered saline (PBS) 3 times followed by fixation with $4 \%$ paraformaldehyde for $15 \mathrm{~min}$, then washed 3 times with PBS. Hoechst 33258 was subsequently added at a final concentration of $10 \mu \mathrm{g} / \mathrm{ml}$ in the dark. Thirty minutes later, the cells were carefully washed twice with PBS. The apoptotic cells were examined and identified according to the condensation and fragmentation of their nuclei under a fluorescence microscope (Eclipse TE2000-S; Nikon, Tokyo, Japan).

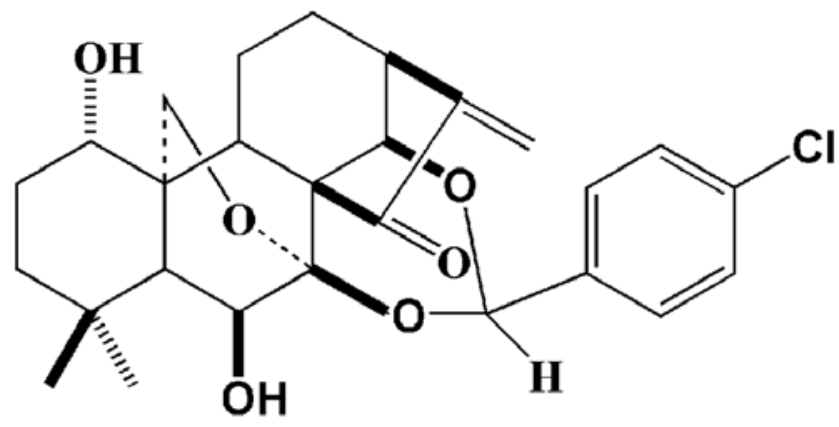

Figure 1. Chemical structure of Jesridonin (JD).

Cell cycle and apoptosis analysis by flow cytometry. To determine the effect of JD on the cell cycle, 5.0x10 ${ }^{6}$ EC109/Taxol cells in the exponential growth phase were treated with various concentrations of JD for $24 \mathrm{~h}$. After an incubation period, the cells were collected, centrifuged and fixed with ice-cold $70 \%$ ethanol at $4^{\circ} \mathrm{C}$ overnight. The cells were washed with PBS before staining with PI and then suspended in staining buffer $(50 \mu \mathrm{g} / \mathrm{ml}$ PI, $1 \%$ Triton X-100 and $0.5 \mathrm{mg} / \mathrm{ml}$ RNase A in PBS) at $37^{\circ} \mathrm{C}$ for $30 \mathrm{~min}$ in the dark. The cells were analyzed on an Accuri C6 flow cytometer (Becton-Dickinson, Franklin Lakes, NJ, USA). The histograms of DNA distribution were shown as a sum of cell populations at the G0/G1, G2/M or $\mathrm{S}$ phase using FlowJo software.

An Annexin V-fluorescein isothiocyanate kit (Annexin VFITC; Nanjing KeyGen Biotech Co., Ltd., Nanjing, China) was used to stain and identify the percentage of apoptotic cells. Briefly, the cells were seeded into 6-well plates and treated with $0,10,20$ and $40 \mu \mathrm{M}$ of JD for $24 \mathrm{~h}$. The cells were then harvested, washed with ice-cold PBS and resuspended in binding buffer containing Annexin V-FITC $(0.5 \mathrm{mg} / \mathrm{ml})$ and PI $(0.5 \mathrm{mg} /$ $\mathrm{ml})$. Following $15 \mathrm{~min}$ of incubation at room temperature in the dark, the stained cells were analyzed immediately by an Accuri C6 flow cytometer (Becton-Dickinson). Apoptotic cells were determined by cells that were both Annexin V-positive and PI-negative.

Protein extraction and western blot analysis. Western blot analyses were conducted as described previously (8). The blots were then probed with each of the following antibodies: anti-p53 antibody (1:1,000), anti-Bcl-2 antibody (1:500), anti-Bax antibody $(1: 1,000)$, anti-PUMA antibody (1:500), anti-procaspase-3 antibody $(1: 1,000)$, anti-cleaved-caspase-3 antibody (1:250) and anti-procaspase- 9 antibody $(1: 1,000)$. After washing the membrane with PBST (PBS, 0.05\% Tween-20) 3 times (10 min each), the blots were subsequently incubated with a goat antimouse or anti-rabbit IgG-HRP secondary antibody $(1: 10,000)$ at $37^{\circ} \mathrm{C}$ for $2 \mathrm{~h}$. The antibody-reactive bands were revealed by an enhanced chemiluminescence substrate and were exposed to Kodak radiographic film.

Statistical analysis. All statistical analyses were carried out using the SPSS 17.0 statistical packages. The data are expressed as the means \pm standard deviation (SD). Continuous variables were analyzed using the Student's t-test. A value of $\mathrm{P}<0.05$ was considered to indicate a statistically significant difference, and a value of $\mathrm{P}<0.01$ a highly statistically significant difference. 

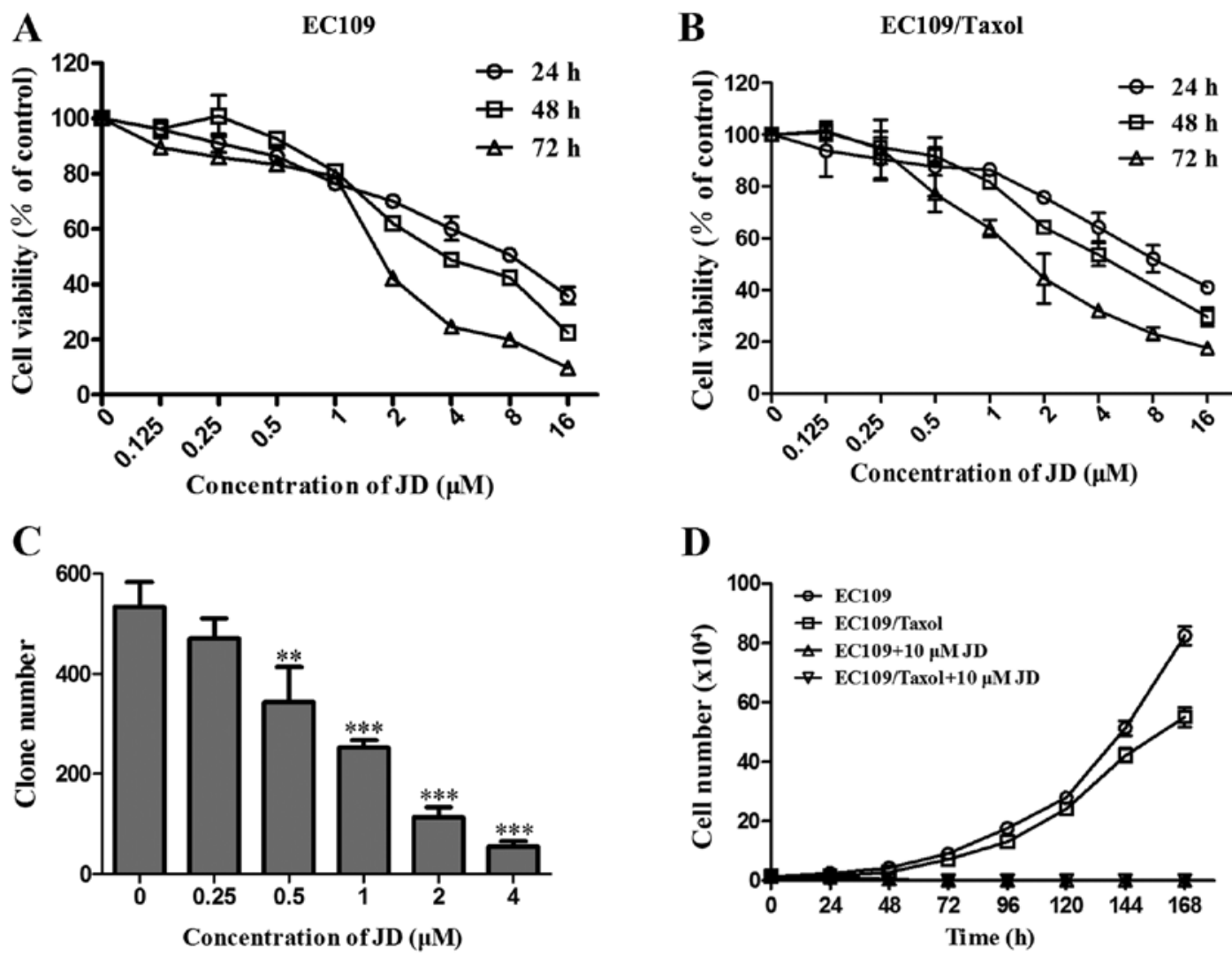

Figure 2. Effect of Jesridonin (JD) on EC109/Taxol and EC109 cell proliferation. (A) EC109 and (B) EC109/Taxol cells were treated with JD at the indicated concentrations for 24,48 and $72 \mathrm{~h}$. Cell viability was determined by MTT assay. Culture medium with $0.1 \%$ dimethyl sulfoxide (DMSO) was used as a control. (C) Colony formation assays were performed to determine the effects of JD treatment on the colony-forming ability of EC109/Taxol cells. (D) The effects of JD on EC109 and EC109/Taxol cell proliferation curves. ${ }^{* *} \mathrm{P}<0.01$ and ${ }^{* * * * *} \mathrm{P}<0.001$ vs. control. The data are shown as the means $\pm \mathrm{SD}$.

Table II. Inhibitory effects of JD on the colony formation of EC109/Taxol cells.

\begin{tabular}{lc}
\hline Group & Colony formation rate $(\%)$ \\
\hline EC109/Taxol & $53.30 \pm 0.04$ \\
$0.25 \mu \mathrm{M}$ JD & $47.00 \pm 0.04$ \\
$0.5 \mu \mathrm{M} \mathrm{JD}$ & $34.33 \pm 0.07^{\mathrm{a}}$ \\
$1 \mu \mathrm{M}$ JD & $25.24 \pm 0.01^{\mathrm{a}}$ \\
$2 \mu \mathrm{M}$ JD & $11.36 \pm 0.02^{\mathrm{b}}$ \\
$4 \mu \mathrm{M}$ JD & $0.06 \pm 0.01^{\mathrm{b}}$ \\
\hline
\end{tabular}

Data are the means \pm SD of 3 independent experiments. ${ }^{\mathrm{a}}<0.01 \mathrm{vs}$. EC109/Taxol cell group; ${ }^{\text {P}} \mathrm{P}<0.001$ vs. EC109/Taxol cell group. JD, Jesridonin.

\section{Results}

Inhibition of cancer cell growth in vitro. Our initial experiment was carried out to determine the inhibitory effect of JD on EC109 and EC109/Taxol cells by MTT assay. The results revealed that JD displayed similar $\mathrm{IC}_{50}$ values against drugsensitive EC109 cells and paclitaxel-resistant EC109/Taxol cells in vitro and indicated that JD had a potent growth inhibitory effect on both of these cell lines in a concentration- and time-dependent manner (Fig. 2A and B and Table I).
The activity of JD was further evaluated using the colony formation assay. As shown in Fig. 2C and Table II, JD not only significantly inhibited the proliferation of EC109/Taxol cells, but also reduced the colony-forming ability compared with the control groups. The colony formation assays demonstrated that JD treatment at concentrations as low as $0.5 \mu \mathrm{M}$ significantly suppressed the colony-forming activity of EC109/Taxol cells. According to the inhibitory effects of JD on the colonyformaing ability of EC109/Taxol cells (Table II), we calculated that the $\mathrm{IC}_{50}$ value of JD was $0.87 \mu \mathrm{M}$ using SPSS software. The data also indicated that the JD-induced suppression of colony formation correlated well with the concentrations of JD, suggesting that JD treatment inhibited the colony-forming ability of EC109/Taxol cells in a dose-dependent manner.

In addition, proliferation curves were generated for the EC109/Taxol and EC109 cells incubated with $10 \mu \mathrm{M}$ of JD. The results indicated that $10 \mu \mathrm{M}$ JD induced significantly decreased the proliferation of EC109/Taxol and EC109 cells. After $48 \mathrm{~h}$ and $72 \mathrm{~h}$ of incubation, the numbers $\left(\mathrm{x} 10^{4}\right)$ of the EC109/Taxol (48 h-0.219 $\pm 0.03,72$ h- $0.125 \pm 0.01)$ and EC109 (48 h-0.563 $\pm 0.02,72 \mathrm{~h}-0.025 \pm 0.01$ ) cells were only a few. Following $96 \mathrm{~h}$ of incubation, the proliferation of the EC109/ Taxol and EC109 cells was completely inhibited (Fig. 2D).

Assessment of cancer growth in mice. To evaluate the in vivo protective effects of JD, we used the growth of EC109/Taxol cell xenografts in female nude mice as an in vivo model. Five animals per treatment group, injected intravenously, were used. 


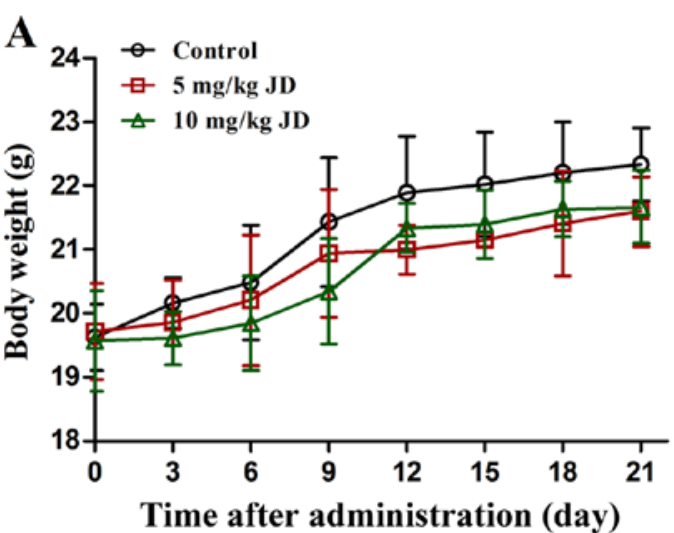

C

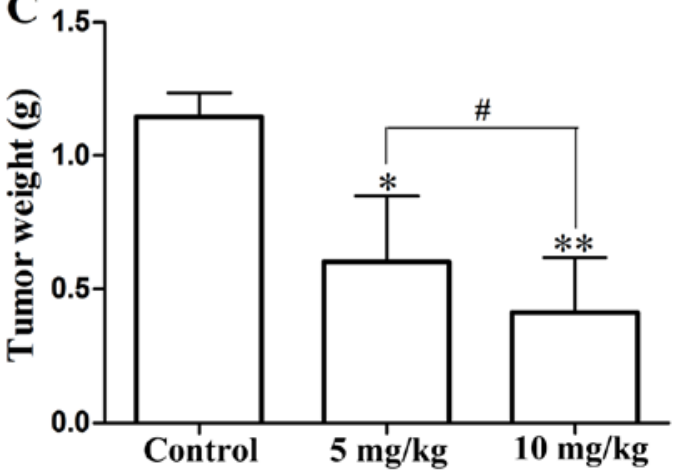

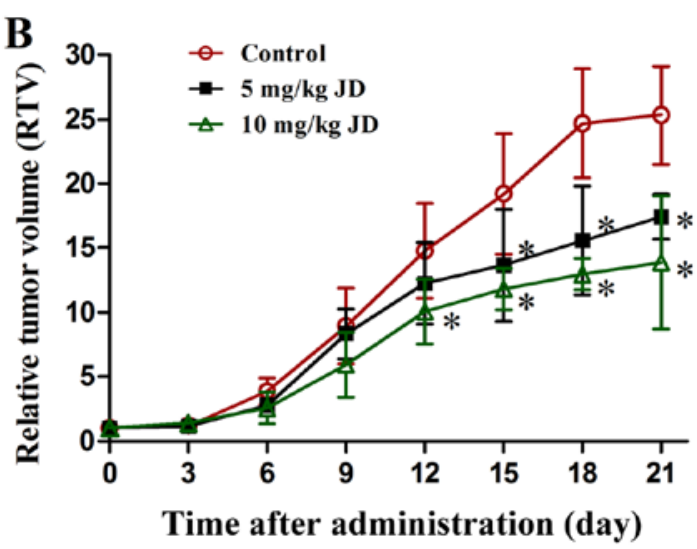

D

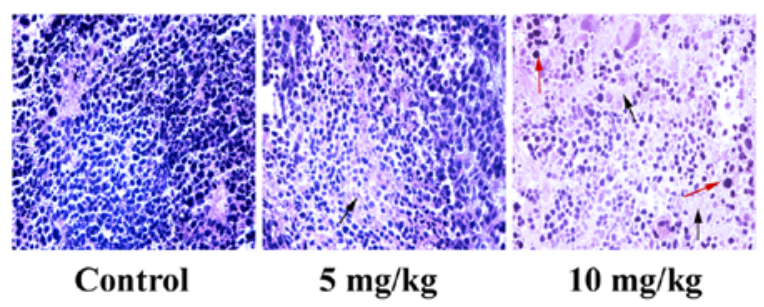

Figure 3. In vivo antitumor effects of Jesridonin (JD) in EC109/Taxol cell-bearing nude mice. EC109/Taxol cells were transplanted subcutaneously into BALB-C nude mice, which were subjected to saline and JD treatment ( 5 and $10 \mathrm{mg} / \mathrm{kg}$ ) for 21 days and then analyzed for tumor relative tumor volume (RTV). (A) The body weights of mice with the indicated treatments. (B) Tumor growth curves were constructed by plotting tumor volumes against time. (C) Tumor weights with the indicated treatment. (D) Representative photographs of H\&E staining in xenografts, original magnification, x200. A low cell density (black arrows) and multinucleated cells and pyknosis (red arrows) indicate mitotic catastrophe and apoptosis. ${ }^{*} \mathrm{P}<0.05$ and ${ }^{* *} \mathrm{P}<0.01 \mathrm{vs}$. the control group; ${ }^{*} \mathrm{P}<0.05$ was considered as statistically significant. The data are shown as the means \pm SD.

Table III. Percentage of cells in the different cell cycle phases.

\begin{tabular}{lccc}
\hline Group & G0/G1 (\%) & $\mathrm{S}(\%)$ & $\mathrm{G} 2 / \mathrm{M}(\%)$ \\
\hline EC109/Taxol & $47.96 \pm 0.91$ & $26.48 \pm 1.24$ & $25.56 \pm 2.02$ \\
$10 \mu \mathrm{M} \mathrm{JD}$ & $43.58 \pm 0.87^{\mathrm{a}}$ & $22.65 \pm 1.56^{\mathrm{a}}$ & $33.95 \pm 1.97^{\mathrm{a}}$ \\
$20 \mu \mathrm{M} J \mathrm{C}$ & $35.23 \pm 2.45^{\mathrm{a}}$ & $15.27 \pm 0.96^{\mathrm{a}}$ & $48.29 \pm 1.38^{\mathrm{a}}$ \\
$40 \mu \mathrm{M} J \mathrm{a}$ & $18.61 \pm 2.14^{\mathrm{a}}$ & $11.25 \pm 0.98^{\mathrm{a}}$ & $69.10 \pm 4.02^{\mathrm{a}}$ \\
\hline
\end{tabular}

The results are means $\pm \mathrm{SD}$ of 3 different experiments. ${ }^{\mathrm{a}} \mathrm{P}<0.01$ indicates significance compared with the EC109/Taxol cell group. JD, Jesridonin.

No significant difference was observed in the body weight changes among the different treatment groups, suggesting that this regimen was safe (Fig. 3A). Compared with the control group, the group treated with JD (either 5 or $10 \mathrm{mg} / \mathrm{kg}$ ) demonstrated a significantly inhibition of tumor growth, both in terms of tumor size and weight (Fig. 3B and C).

Histological analysis of the H\&E-stained tumor sections from the EC109/Taxol xenografts from the mice treated with JD demonstrated a marked change in tissue and cell morphology compared with those from the vehicle control group (Fig. 3D). These changes included a low cell density and multinucleated cells with condensed chromatin staining and pyknosis, indicating mitotic catastrophe and apoptosis.

Effects of JD on the cell cycle distribution of EC109/Taxol cells. The cellular DNA content of JD-treated cells and untreated cells was analyzed by flow cytometry to detect changes in the cell cycle distribution of the EC109/Taxol cells. As shown in Fig. 4 and Table III, treatment of the EC109/Taxol cells with JD at 10, 20 and $40 \mu \mathrm{M}$ for $24 \mathrm{~h}$ significantly increased the $\mathrm{G} 2 / \mathrm{M}$ cell population up to $33.95 \pm 1.97,48.29 \pm 1.38$ and $69.169 .10 \pm 4.02 \%$, respectively, whereas $25.56 \pm 2.02 \%$ of the control cell population was in the G2/M phase. The increase in the G2/M cell population induced by JD occurred at the expense of a decrease in the G0/G1 and $\mathrm{S}$ cell populations. These results indicated that the cells treated with JD exhibited a G2/M phase arrest.

JD induces the apoptosis of EC109/Taxol cells. Following treatment with JD at the indicated concentrations for $24 \mathrm{~h}$, morphological changes in EC109/Taxol cells were assessed using an inverted microscope. DMSO $(0.1 \%)$ was used as a control. The EC109/Taxol cells treated with JD exhibited apoptosis-related morphologies, such as cell shrinkage and rounding up, cell membrane blebbing and nuclear fragmentation and condensation (Fig. 5A). In addition, nuclear morphological changes at the corresponding concentration were also recorded and qualitatively evaluated by means of Hoechst 33258 fluorescent staining following incubation for $24 \mathrm{~h}$. Typical apoptotic 


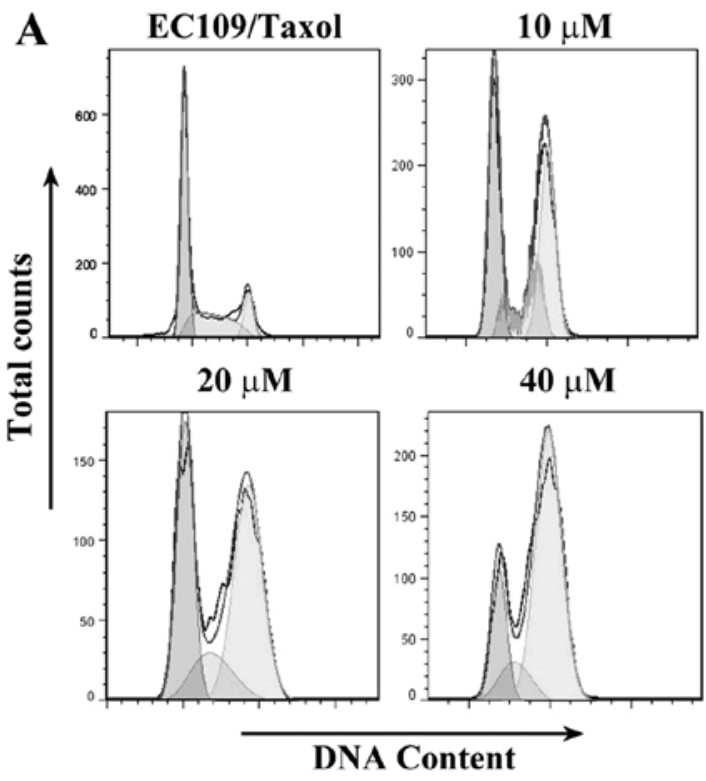

B

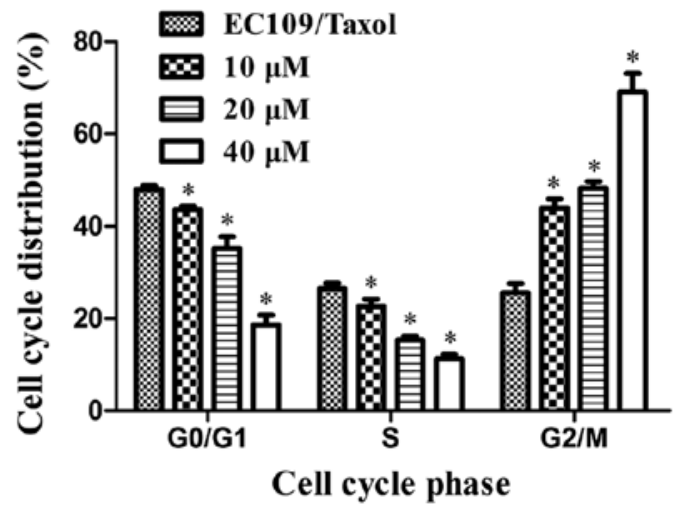

Figure 4. Jesridonin (JD) induces the accumulation of the G2/M cell population in EC109/Taxol cells. (A) Flow cytometric determination of DNA contents was analyzed using an Accuri C6 flow cytometer. (B) The cell cycle distribution among the different treatment groups are presented in percentages as a histogram graph. The experiments were repeated 3 times, and the data are shown as the means $\pm \mathrm{SD}$. ${ }^{*} \mathrm{P}<0.01$ indicates significance compared with the EC109/Taxol cell group.

markers, including nuclear fragmentation and nuclear condensation, were detected, particularly at the highest concentrations of JD (Fig. 5B).

In addition, to determine whether JD treatment induces apoptosis, cells incubated with increasing concentrations of JD for $24 \mathrm{~h}$ were analyzed using flow cytometry to assess the diploid DNA content and for phosphatidylserine (PS) exposure to the cell surface. The percentages of live, early-apoptotic, late-apoptotic and necrotic cells were calculated using an Accuri C6 flow cytometer. Treatment of the EC109/Taxol cells for $24 \mathrm{~h}$ with 10,20 and $40 \mu \mathrm{M}$ of JD resulted in a dose-dependent increase in the percentage of the apoptotic population up to $6.7 \pm 1.80,36.2 \pm 3.60$ and $46.1 \pm 2.62 \%$, respectively, compared with the control treated cells $(3.6 \pm 0.64 \%)$ (Fig. 5C and D).

Western blot analysis. As p53 is a major tumor suppressor, we examined the role of p53 in JD-mediated growth inhibition. JD treatment resulted in an increase in the $\mathrm{p} 53$ protein levels in the EC109/Taxol cells (Fig. 6A and B). Apoptosis is a well-organized cellular event, characterized by the activation of caspase cascades. Caspases are synthesized as pro-enzymes that must be activated to perform their functions. Caspase-9 is the major caspase member that regulates the intrinsic apoptosis pathway, whereas caspase- 3 is one of the critical effector caspases in the downstream execution of apoptosis $(5,15)$. As shown in Fig. 6A, F and G, upon treatment with JD, the procaspase-9 and procaspase- 3 levels decreased, and the cleaved-caspase- 9 and cleaved caspase- 3 levels increased, indicating the activation of caspase- 9 and caspase- 3 .

We also examined the expressions levels of Bax and Bcl-2 and then analyzed the ratio of $\mathrm{Bax} / \mathrm{Bcl}-2$. The results of western blot analysis revealed that Bcl-2 expression was significantly decreased and that Bax expression was not altered in the JD-treated EC109/Taxol cells (Fig. 6A and C). Statistical analysis also indicated that JD in the range of $10-40 \mu \mathrm{M}$ increased the ratio of Bax/Bcl-2 (Fig. 6D). Moreover, PUMA expression was upregulated following treatment with JD (Fig. 6A and E).

\section{Discussion}

The EC109/Taxol cells, a paclitaxel-resistant cell line expressing high levels of P-gp, were generated from the drugsensitive parental EC109 cells by the intermittent exposure to a high concentration of PTX in stepwise time increments over a period of 6 months. The PTX resistance of the EC109/ Taxol cell line was approximately 67.2-fold that of the EC109 cell line. In addition, the EC109/Taxol cells exhibited a greater cross-resistance to 5-fluorouracil (5-FU), cisplatin (CDDP) and epirubicin (EPI) than its parental EC109 cell line (8). In this study, we examined the effect of JD on EC109/Taxol cells both in vitro and in vivo. JD exhibited a strong activity against EC109/Taxol cells. The JD-induced inhibition of cancer growth was confirmed in nude mouse models. The results indicated that EC109/Taxol xenografts were sensitive to JD.

Cell cycle perturbation plays an important role in carcinogenesis, and cell proliferation, apoptosis, differentiation and senescence are all cell cycle-dependent (16). In the next set of experiments, we examined the effects of JD on the cell cycle distribution of EC109/Taxol cells. EC109/Taxol cells were treated with JD at various concentrations $(0,10,20$ and $40 \mu \mathrm{M})$ for $24 \mathrm{~h}$. JD caused an accumulation of cells in the G2/M phase and diminished cells in the S and G0/G1 phases compared with the untreated control cells. The results suggested that JD caused an obvious $\mathrm{G} 2 / \mathrm{M}$ arrest pattern in a concentration-dependent manner, with a concomitant decrease in terms of the number of cells in other phases of the cell cycle.

Apoptosis is a regulated type of physiological cell death in which a controlled sequence of events leads to cell elimination essential for the maintenance of homeostasis $(17,18)$. As the death of tumor cells by chemotherapy is mediated mainly by 
A
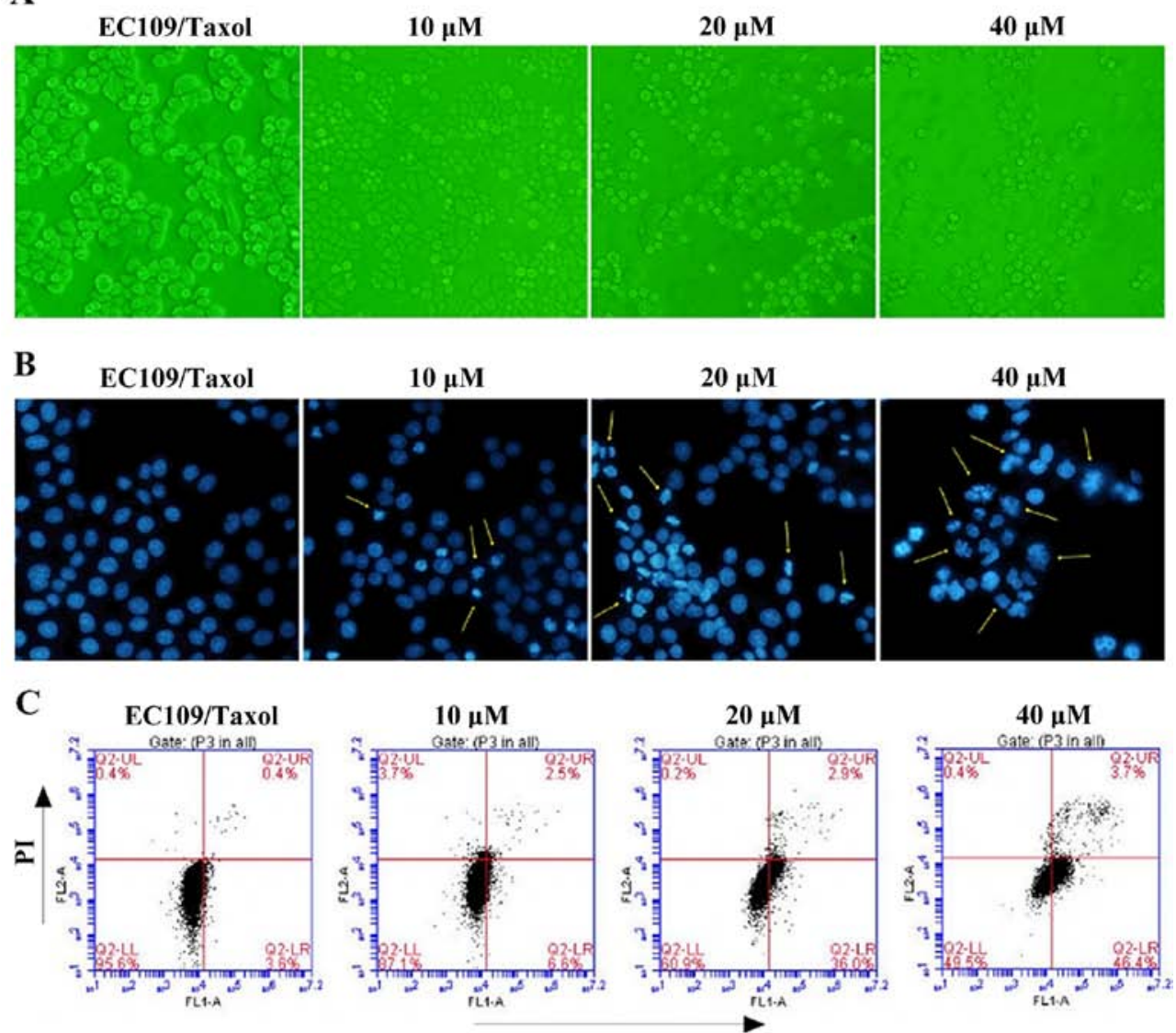

Annexin V-FITC

D

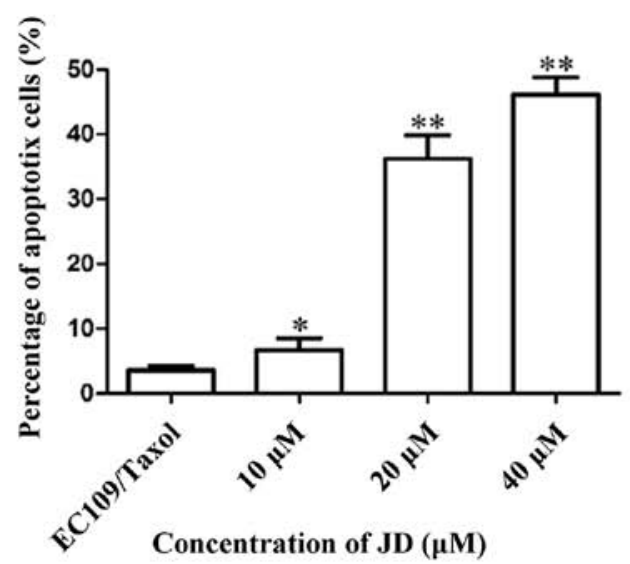

Figure 5. Apoptosis was analyzed by flow cytometry. EC109/Taxol cells treated with Jesridonin (JD) exhibited typical apoptotic morphologies. (A) Morphological changes in EC109/Taxol cells treated with JD for $24 \mathrm{~h}$ at the indicated concentrations (magnification, x200). (B) Effects of JD on the nuclear morphology of EC109 cells were assessed via Hoechst 33258 staining (magnification, x200). The arrow heads indicate the condensation of chromatin material and fragmentation of nuclei in apoptotic cells. (C) Cell apoptosis was evaluated by Annexin V-propidium iodide (PI) staining and then analyzed using flow cytometry. A representative image is shown. The analysis standard was as follows: Annexin-V-negative and PI-negative cells were identified as normal cells; Annexin-Vpositive and PI-negative cells were identified as early apoptotic cells; and Annexin-V-positive and PI-positive cells were identified as late apoptotic cells. (D) The percentages of apoptotic cells are summarized in the histogram graphs. The experiments were repeated 3 times, and the results are presented as the means \pm SD. ${ }^{*} \mathrm{P}<0.05$ and $^{* *} \mathrm{P}<0.01$ vs. the control group.

the induction of apoptosis, the deregulation of apoptosis would confer resistance to anticancer agents. In this study, apoptosis, as determined using Hoechst 33258 staining and flow cytometric analysis (Fig. 5), was confirmed to be responsible for the JD-mediated anti-resistance activity in EC109/Taxol cells. Thereby, our research provides new approaches to overcoming drug resistance by inducing apoptosis in resistant EC109/Taxol cell lines.
For further study, we elucidated the possible mechanisms responsible for the apoptosis induced by JD. Although the mechanism of drug resistance is not completely defined, the deficiency in apoptosis and abnormally activated survival signaling pathways have been observed in most cancers with MDR. Apoptosis deficiency may be involved in cancer initiation, progression and treatment failure in cancers with MDR (19). Apoptosis is regulated at multiple levels 

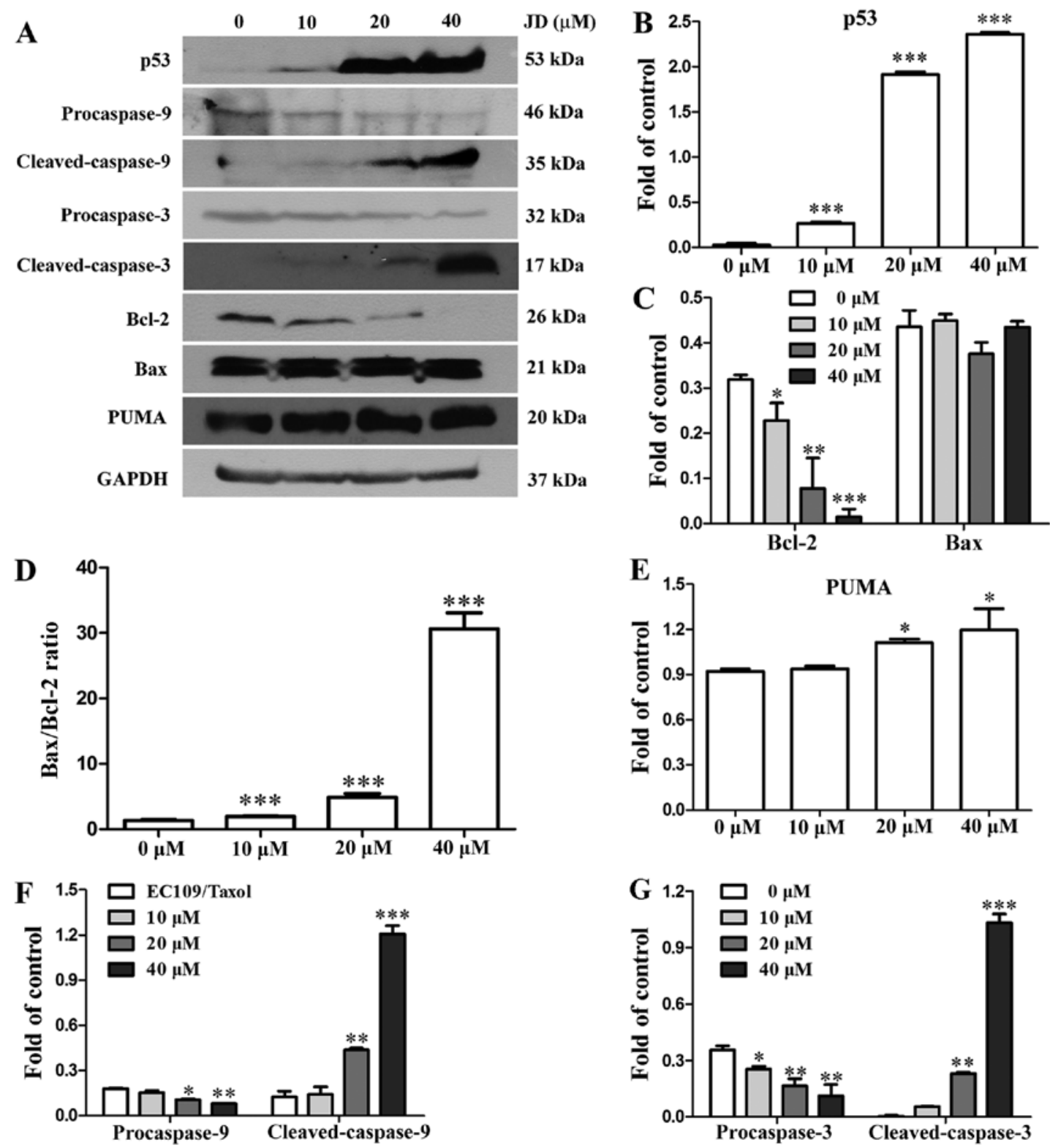

Figure 6. Western blot analysis for the detection of pro-apoptotic and anti-apoptotic proteins. (A) Expression of p53, Bcl-2, Bax, PUMA procaspase-9, cleaved-caspase-9, procaspase-3 and cleaved-caspase-3 in EC109/Taxol cells treated with different concentrations of Jesridonin (JD) for 24 h. (B-G) Statistical analysis of the western blots. All data represent the means $\pm \mathrm{SD}$ of 3 independent experiments. ${ }^{*} \mathrm{P}<0.05,{ }^{* *} \mathrm{P}<0.01$ and ${ }^{* * *} \mathrm{P}<0.001$ vs. control.

in the extrinsic and intrinsic pathways (20). The extrinsic pathway involves death receptors on the cell surface that can directly activate caspase- 8 . The intrinsic pathway of apoptosis centers on the mitochondria and is associated with the loss of the mitochondrial membrane potential (MMP), the release of cytochrome $c$ into the cytosol and the activation of caspase-9/3 $(21,22)$. The Bcl-2 and caspase families are considered as the most important proteins for regulating apoptosis in the intrinsic pathway. The Bcl-2 family can be divided into two types: anti-apoptotic and pro-apoptotic proteins (23). The ratio of $\mathrm{Bcl}-2 / \mathrm{Bax}$ is a critical determinant for cell apoptosis. The dysregulation of the Bcl-2 family has been shown to induce the destruction of the mitochondrial membrane, which is accompanied by the release of intramembranous proteins into the cytosol, such as cytochrome $c$ and other apoptosis-inducing factors, and to subsequently induce the activation of the caspase cascade by activating caspase- 9 and caspase- $3(24,25)$. Bax is constitutively located within the cytosol, whereas the activation of Bax involves its translocation from the cytosol to the outer mitochondrial membrane and, subsequently, its insertion into the membrane (26). Bax can release cytochrome $c$ after the formation of the Bax/Bak hetero-oligomer, whereas Bcl-2 can interact with activator proteins or Bax/Bak, thereby sequestering these proteins (27-29). The BH3-only protein PUMA is a pro-apoptotic protein that is normally expressed at a low level. Its upregulation leads to cytochrome $c$ release, which results in apoptosis (30). The tumor suppressor and transcription factor p53 is a key modulator of cellular stress responses, and the activation of p53 can trigger apoptosis in many cell types $(31,32)$. Therefore, the dysregulation of the Bcl-2 and caspase families and of p53, as well as their respective signaling pathways, has been considered the most important mechanism in the development of apoptotic deficiency and the phenotype of MDR in cancer cells. The regulation of these protein kinases may be an 
effective regimen for the treatment of cancers with apoptotic deficiency and cells with MDR (33).

Our results indicated that treatment with JD significantly downregulated anti-apoptotic Bcl-2 protein expression, upregulated PUMA expression and did not alter pro-apoptotic Bax protein expression, thus shifting the $\mathrm{Bax} / \mathrm{Bcl}-2$ ratio in favor of apoptosis. Moreover, JD upregulated the expression of p53, cleaved-caspase-9 and cleaved-caspase-3 in EC109/Taxol cells and downregulated the expression of procaspase- 3 and procaspase-9 in EC109/Taxol cells in a concentration-dependent manner. We therefore suggested that the induction of apoptosis in EC109/Taxol cells by JD may be due to the activation of the mitochondria-mediated intrinsic apoptosis pathway. Further research on the specific mechanisms of action and the efficacy of JD in other cancer cell lines is currently underway.

In conclusion, to the best of our knowledge, our results demonstrate for the first time that JD exerts an anti-proliferative and anti-MDR effect on EC109/Taxol cells. JD treatment via intravenous injection in mice effectively prevented the growth of EC109/Taxol xenografts without exerting any significant toxicity. These findings suggest the possibility that JD may be a promising drug candidate and provide new supporting evidence for the manipulation of the dysregulated apoptotic pathway as a strategy with which to overcome drug resistance.

\section{Acknowledgements}

This study was supported by the National Natural Science Foundation of China (project nos. 21372206 and 81430085, for H.L.), the Natural Science Foundation of Henan Province of China (the foundation and frontier technology research program) (project no. 152300410030 for C.W.), the Science and Technology Research Key Project in the Henan province Department of Education (project no. 14B350011 for C.W.) and the Specialized Research Foundation of the Doctoral Program of High College for new teachers (no. 20104101120012 for C.W.).

\section{References}

1. Sui X, Chen R, Wang Z, Huang Z, Kong N, Zhang M, Han W, Lou F, Yang J, Zhang Q, et al: Autophagy and chemotherapy resistance: a promising therapeutic target for cancer treatment Cell Death Dis 4: e838, 2013.

2. Lu HP and Chao CC: Cancer cells acquire resistance to anticancer drugs: an update. Biomed J 35: 464-472, 2012.

3. Ambudkar SV, Dey S, Hrycyna CA, Ramachandra M, Pastan I and Gottesman MM: Biochemical, cellular, and pharmacological aspects of the multidrug transporter. Annu Rev Pharmacol Toxicol 39: 361-398, 1999.

4. Baguley BC: Multidrug resistance in cancer. Methods Mol Biol 596: 1-14, 2010.

5. Zhou Q, Li Y, Jin J, Lang L, Zhu Z, Fang W and Chen X: Lx2-32c, a novel taxane derivative, exerts anti-resistance activity by initiating intrinsic apoptosis pathway in vitro and inhibits the growth of resistant tumor in vivo. Biol Pharm Bull 35: 2170-2179, 2012.

6. Mor G, Montagna MK and Alvero AB: Modulation of apoptosis to reverse chemoresistance. Methods Mol Biol 414: 1-12, 2008

7. Hsu PC, Hung HC, Liao YF, Liu CC, Tsay GJ and Liu GY: Ornithine decarboxylase attenuates leukemic chemotherapy drugs-induced cell apoptosis and arrest in human promyelocytic HL-60 cells. Leuk Res 32: 1530-1540, 2008.

8. Wang C, Guo LB, Ma JY, Li YM and Liu HM: Establishment and characterization of a paclitaxel resistant human esophageal carcinoma cell line. Int J Oncol 43: 1607-1617, 2013

9. Liu Z, Ouyang L, Peng H and Zhang WZ: Oridonin: Targeting programmed cell death pathways as an anti-tumour agent. Cell Prolif 45: 499-507, 2012.
10. Li CY, Wang EQ, Cheng Y and Bao JK: Oridonin: An active diterpenoid targeting cell cycle arrest, apoptotic and autophagic pathways for cancer therapeutics. Int J Biochem Cell Biol 43: 701-704, 2011.

11. Wang C, Jiang L, Wang S, Shi H, Wang J, Wang R, Li Y, Dou Y, Liu Y, Hou G, et al: The antitumor activity of the novel compound Jesridonin on human esophageal carcinoma cells. PLoS One 10: $\mathrm{e} 0130284,2015$.

12. Tian QE, Li HD, Yan M, Cai HL, Tan QY and Zhang WY: Astragalus polysaccharides can regulate cytokine and P-glycoprotein expression in H22 tumor-bearing mice. World J Gastroenterol 18: 7079-7086, 2012.

13. Wacheck V and Zangemeister-Wittke U: Antisense molecules for targeted cancer therapy. Crit Rev Oncol Hematol 59: 65-73, 2006.

14. Uchiyama-Kokubu N and Watanabe T: Establishment and characterization of adriamycin-resistant human colorectal adenocarcinoma HCT-15 cell lines with multidrug resistance. Anticancer Drugs 12: 769-779, 2001.

15. Fulda $\mathrm{S}$ and Debatin KM: Extrinsic versus intrinsic apoptosis pathways in anticancer chemotherapy. Oncogene 25: 4798-4811, 2006.

16. Zhang J, Zhu X, Li H, Li B, Sun L, Xie T, Zhu T, Zhou H and Ye Z: Piperine inhibits proliferation of human osteosarcoma cells via $\mathrm{G} 2 / \mathrm{M}$ phase arrest and metastasis by suppressing MMP-2/-9 expression. Int Immunopharmacol 24: 50-58, 2015.

17. Hafezi F, Steinbach JP, Marti A, Munz K, Wang ZQ, Wagner EF, Aguzzi A and Remé CE: The absence of c-fos prevents light-induced apoptotic cell death of photoreceptors in retinal degeneration in vivo. Nat Med 3: 346-349, 1997.

18. Cao B, Chen H, Gao Y, Niu C, Zhang Y and Li L: CIP-36, a novel topoisomerase II-targeting agent, induces the apoptosis of multidrug-resistant cancer cells in vitro. Int J Mol Med 35: 771-776, 2015.

19. Uboldi S, Bernasconi S, Romano M, Marchini S, Fuso Nerini I, Damia G, Ganzinelli M, Marangon E, Sala F, Clivio L, et al: Characterization of a new trabectedin-resistant myxoid liposarcoma cell line that shows collateral sensitivity to methylating agents. Int J Cancer 131: 59-69, 2012.

20. Bai HW, Badaboina S, Park CH, Choi BY, Na YH and Chung BY: Centipedegrass extract induces apoptosis through the activation of caspases and the downregulation of PI3K/Akt and MAPK phosphorylation in leukemia cells. Int J Mol Med 35: 511-518, 2015.

21. Kroemer G and Reed JC: Mitochondrial control of cell death. Nat Med 6: 513-519, 2000.

22. Fares M, Abedi-Valugerdi M, Hassan M and Potácová Z: DNA damage, lysosomal degradation and $\mathrm{Bcl}-\mathrm{xL}$ deamidation in doxycycline- and minocycline-induced cell death in the K562 leukemic cell line. Biochem Biophys Res Commun 463: 268-274, 2015.

23. Peng J, Ding J, Tan C, Baggenstoss B, Zhang Z, Lapolla SM and Lin J: Oligomerization of membrane-bound Bcl-2 is involved in its pore formation induced by tBid. Apoptosis 14: 1145-1153, 2009.

24. Kluck RM, Bossy-Wetzel E, Green DR and Newmeyer DD: The release of cytochrome $c$ from mitochondria: A primary site for Bcl-2 regulation of apoptosis. Science 275: 1132-1136, 1997.

25. Degli Esposti M and Dive C: Mitochondrial membrane permeabilisation by Bax/Bak. Biochem Biophys Res Commun 304: 455-461, 2003.

26. Chipuk JE, Kuwana T, Bouchier-Hayes L, Droin NM, Newmeyer DD, Schuler M and Green DR: Direct activation of Bax by 53 mediates mitochondrial membrane permeabilization and apoptosis. Science 303: 1010-1014, 2004.

27. Kang MH and Reynolds CP: Bcl-2 inhibitors: Targeting mitochondrial apoptotic pathways in cancer therapy. Clin Cancer Res 15: 1126-1132, 2009.

28. Bodur $\mathrm{C}$ and Basaga $\mathrm{H}$ : Bcl-2 inhibitors: Emerging drugs in cancer therapy. Curr Med Chem 19: 1804-1820, 2012.

29. Nikoletopoulou V, Markaki M, Palikaras K and Tavernarakis N: Crosstalk between apoptosis, necrosis and autophagy. Biochim Biophys Acta 1833: 3448-3459, 2013.

30. Seervi M, Sobhan PK, Joseph J, Ann Mathew K and Santhoshkumar TR: EROl $\alpha$-dependent endoplasmic reticulummitochondrial calcium flux contributes to ER stress and mitochondrial permeabilization by procaspase-activating compound-1 (PAC-1). Cell Death Dis 4: e968, 2013.

31. Culmsee $C$ and Mattson MP: p53 in neuronal apoptosis. Biochem Biophys Res Commun 331: 761-777, 2005.

32. Tacar O and Dass CR: Doxorubicin-induced death in tumour cells and cardiomyocytes: Is autophagy the key to improving future clinical outcomes? J Pharm Pharmacol 65: 1577-1589, 2013.

33. West KA, Castillo SS and Dennis PA: Activation of the PI3K/Akt pathway and chemotherapeutic resistance. Drug Resist Updat 5: 234-248, 2002. 